\title{
Assessment of cognitive impairment by using Addenbrooke's Cognitive Examination (ACE) and Montreal Cognitive Assessment (MoCA) amongst type 2 diabetes mellitus patients in Eastern Uttar Pradesh, India
}

\author{
S. Kant ${ }^{1, *}$, Karan Poddar ${ }^{2}$, M. Kamle ${ }^{3}$, C. Patil ${ }^{4}$ \\ Consultant, ${ }^{1,2}$ Neurology Centre, Varanasi, Uttar Pradesh, ${ }^{3}$ Kamle Clinic, Vikroli, Mumbai, Maharashtra, Vardhman Clinic, \\ Thane, Maharashtra, India
}

*Corresponding Author:

Email: drskpoddar@gmail.com

\begin{abstract}
Aim: The occurrence of diabetes mellitus (DM) is in increasing trend worldwide predominantly in South East Asian countries comprising India. Also, there is strong evidences that, DM upsurges the risk of cognitive impairment and dementia. Hence our main aim behind this study was to divulge the relationship between DM and mild cognitive impairment (MCI).

Materials and Methods: A retrospective cohort study was conducted by reviewing and analyzing the medical records of DM who had been consulted at the neurology out-patient department during a period of $2015-2016$.

The cognitive impairment in DM patients were assessed by using Addenbrooke's Cognitive Examination (ACE) and Montreal Cognitive Assessment (MoCA).

Statistical Analysis: The data was analyzed using Statistical Package for Social Sciences (SPSS) version 19 software. If the pvalue $<0.05$ was regarded as statistically significant.

Results: The prevalence of cognitive impairment in DM patient is seemingly high with both scores i.e. $96.31 \%$ with MoCA and 99.85\% with ACE-R. The variables like sex and age groups were not statistically significant with MoCA scores. The MoCA score \& ACE-R score showed a negative association with education levels. The MoCA scores of different items i.e. visuoexcutive, naming, attention, language, abstraction and orientation were highly statistically significant at $0.01 \%$ level, between the DM patients who had cognitive impairment and DM patients with normal score, except memory which score zero in all patients. Conclusion: The prevalence of cognitive impairment in DM patients is very high. The, elder age, low education attainment, and unskilled occupation were also the potential identified risk factors for cognitive impairment.
\end{abstract}

Keywords: Diabetes, Dementia, MoCA, Cognitive impairment, Low education etc.

\section{Introduction}

The prevalence of diabetes mellitus (DM) is increasing worldwide; currently it is assuming pandemic status. According to the International Diabetes Federation (IDF) Atlas guideline report $\left(8^{\text {th }}\right.$ edition), currently there are 425 million people have diabetes in the world and 82 million people in the South East Asia region; by 2045 this will rise to 151 million. There were over 72,946,400 cases of diabetes in India in $2017 .{ }^{1}$

In India's utmost population is representing the rural area, there were several studies done for the prevalence of DM but most of them were conducted in urban setting. However, the enormous majority of India's population $(70 \%)$ lives in rural areas. ${ }^{2}$ Screening for DM is seldom done in rural areas, resulting in a much greater burden of undiagnosed DM in rural areas. The published literatures claimed that, in Eastern Utter Pradesh the DM moved from 37th to 15 th position in a ranking of leading causes of death and disability in the duration of 1990 to $2016 .{ }^{3}$ Therefore the present study was undertaken to find out the prevalence and complications of DM in rural area of Eastern Utter Pradesh, India. DM has overwhelming effects on older adults; adults with DM might have increased risk for developing micro and macro vascular complications (e.g., retinopathy, neuropathy, and cardiovascular disease). ${ }^{4}$ As literatures supported, DM is the most complex disease and many organ systems are unfavorably affected by DM, including the brain, which undergoes changes that may increase the risk of cognitive decline (as in memory, attention, coordination, and psychomotor speed). ${ }^{5}$

Several research studies have emphasized that DM is associated with higher risk of cognitive dysfunction, dementia, and depression in the elderly patients. ${ }^{6}$ The root pathogenesis of cognitive impairment and depression in DM is multifactorial and unidentified. However, it is associated with the decline of cognitive functions and it might be a one of the risk factor for the development of mild cognitive impairment (MCI). MCI is likely to be a possible impermanent stage between normal cognitive function and Alzheimer's disease (AD) which is characterized by subjective memory complaints, objective memory impairment, normal cognitive functioning, intact daily living activities, and absence of dementia. However, when it comes to the conversion rate from MCI to dementia was reported to be $11.65 \%$ per year. $^{7} \mathrm{AD}$ is the most communal type of dementia in the midst of the elderly patients accounting for the almost 50-60\% of elderly patient with dementia. In sustenance with this, recent studies also showed that $\mathrm{DM}$ is robust risk factor in development of AD. ${ }^{8}$ Hence, the high risk of MCI and dementia in DM patients need 
to be observing predictors of cognitive impairment. In India as per routine practice evaluation of cognitive functioning was not been given considerable importance. However, with consideration to increasing life expectancy and increase in DM in India, it's pertinent to evaluate cognitive impairment in DM patient by using a simple screening test. ${ }^{9}$

As per Singh P S et al, the prevalence of DM in the rural population was found to be very high $(8.03 \%)$. The same study also find that the female $(9.91 \%)$ were more prone to the DM as compared to males $(6.79 \%){ }^{10}$

However, Kodl et al showed that the patients with DM have been associated with decreased in psychomotor speed, frontal lobe/executive function, verbal memory, processing speed, complex motor functioning, working memory, immediate recall, delayed recall, verbal fluency, visual retention, and attention. ${ }^{11}$

Though the Indian guidelines are not available for management of cognitive impairments due to DM. In this study, the prevalence of disease complications (cognitive impairment) education, elder age, occupation, gender etc. These factors are the preliminary step to help for deciding for the management of cognitive impairment in DM. Even though much research has been done, but still the patho-physiology underlying this complication is not well understood, and the most suitable methods to make out, treat, and avert cognitive dysfunction in DM have not yet been defined. This study was undertaken with the objectives to assess cognitive impairment in DM patients using Addenbrooke's Cognitive Examination (ACE-R) and Montreal Cognitive Assessment (MoCA).

\section{Materials and Methods}

It is a retrospective cohort study, conducted by reviewing and analyzing medical records of DM patients who had been consulted at the neurology outpatient department, screened, and underwent the following assessment tests during a period of one year from 2015 to 2016.

The MoCA is a cognitive screening instrument developed to detect MCI. It is a simple ten minute paper and pencil test to assess the various cognitive domains; it has seven sub scores; memory (5 points for delayed recall), language (3 points), visuospatial/ executive functions (5 points), abstraction ( 2 points), attention (6 points), naming (3 points) and orientation (6 points). Its validity has been established to detect $\mathrm{MCI}$ in patients with $\mathrm{AD}$ and other pathologies in cognitively impaired patients who scored in the normal range on the Mini-Mental State Examination (MMSE). ${ }^{8,9}$

The total possible score for MoCA screening instrument is 30 points with a score of 26 or more considered as a normal. Depending on the education level of the patients, the MoCA scale scores are adjusted accordingly. ${ }^{8}$ The MoCA has a score $\leq 25$ was found to be the optimal cut-off point for a diagnosis of cognitive impairment. ${ }^{8}$ The patients were further divided into two groups, one subset had score between 18 and 25 (mild cognitive impairment) and the other subset which has score below 18 (mild dementia).

$\mathrm{ACE}$ and its subsequent iterations, ACE-R and ACE-III have proved easy to use, acceptable to patients, and have shown excellent diagnostic utility in identifying dementia and cognitive impairment in a variety of clinical situations. ${ }^{11}$

The ACE-R takes between 12 and 20 min (average 16) to administer and score in a clinical setting. It comprises 5 sub-scores, each one representing one cognitive domain: attention/orientation (18 points), memory (26 points), fluency (14 points), language (26 points) and visuospatial (16 points). ACE-R maximum score is 100 , collectively by the addition of the all domains. ${ }^{13}$

It is scored out of 100 , with a higher score denoting better cognitive function. ${ }^{8}$

\section{Data Analysis}

Descriptive values are expressed as means \pm SD or percentages. The data was analyzed using Statistical Package for Social Sciences (SPSS) version 19 software. The qualitative data were expressed as frequencies and percentages, while the quantitative data were summarized as means and standard deviation. The statistical significance was tested using Chi-square test and p-value $<0.05$ was regarded as statistically significant.

\section{Results \\ Study population characteristics}

In present study a medical records of total $678 \mathrm{DM}$ patients were studied, out of that $229(33.78 \%)$ were females and $449(66.22 \%)$ were males with a mean age of $57.45 \pm 8.26$ years (range 45 and 90 years). The mean values of MoCA score for the study population was 17.21 \pm 4.74 and the mean value for ACE-R score was 52.68 \pm 9.69 . However, 25 (3.68\%) DM patients scored above the cut off 26 with MoCA while one DM patient scored above the cut-off of 83 in Addenbrooke's scores. Hence, this study showed, the prevalence of cognitive impairment in DM patients is outwardly high with both scores i.e. $96.31 \%$ with MoCA and $99.85 \%$ with Addenbrooke's respectively.

Further analysis of variables was done with MoCA scores. A comparison of age with the MoCA showed that in the age group of 45-60 years, $36(5.31 \%)$ diabetic patients had MoCA score above 26 and one diabetic patient had Addenbrooke's scores above 83 . However, the diabetic patients age over 60 had only 12 $(1.77 \%)$ diabetic patient had MoCA score greater than 26 and none of the diabetics patient had Addenbrooke's score above 83, showing that though cognitive impairment is higher in the older group but is not statistically significant (Table 1). 
Although, in present study, total number of males $449(66.22 \%)$ more than females $229(33.77 \%)$ the result showed that, the incidence of dementia was found to be slightly more in females $182(26.84 \%)$ than compared to males $172(25.31 \%)$. Hence, the parameter gender was not statistically significant but odds ratio (OR) showed that MCI \& dementia (combined) is 0.362 in males where in females it was 1.0 (Table 1). Interestingly, as per MoCA score, the dementia was most commonly occurred in housewives $(27.1 \%)$ and mild cognitive impairment commonly found in service personnel (20.2\%). However, ACE-R score showed that housewives $(32.74 \%)$ were more susceptible to MCI, followed by service personnel's (30.97\%).

The MoCA score \& ACE-R score showed a negative association with education levels. As the years of education increased there was significant increase in both the MoCA score and ACE-R score. The mean MoCA score in 180 illiterate populations was $12.73 \pm 2.83$, till schooling (and HSC) was $19.36 \pm 4.01$ and increased significantly in post graduates to a score of 22.13 \pm 3.67 . The mean ACE-R score in illiterate population was $43.89 \pm 6.61$, till schooling (and HSC) $56.40 \pm 7.72$ and increased significantly in post graduates to a score of $61.58 \pm 7.70$ (Table 1).

In this study, the alcohol consumption did not affect any significant influence on the dementia as most of the dementia patients were from the non-alcoholic group. This showed the MoCA scores of different items between the DM patients who had cognitive impairment and normal patients (Table 2). Except memory all other tests like visuo-excutive, naming, attention, language, abstraction, and orientation are statistically significant at $0.01 \%$ level i.e., $\mathrm{P}<0.001$ suggesting the scores decreased in both MCI and dementia patients. In memory all values were zero (0) except one patient where the maximum score was 2 . This was not necessary statistically significant but then all patients scored zero on memory. The table results also depicted that the median score is lowest in patients with mild dementia that in patients with cognitive impairment.

\section{Discussion}

Dementia is one of the utmost and overwhelming diseases of late life; nearly 4.6 million new cases of dementia were identified worldwide every year. The numbers of people who are affected are persistently increasing and have been predicted to double every 20 years, 42 million by 2020 and 81 million by $2040^{14}$. In this study, we studied factors associated with dementia and memory cognition in elderly diabetic patients using MoCA and ACE-R. We compared the parameters likes' age, gender, educational attainment, and alcohol addiction. This study demonstrated that, elder age, low education attainment; unskilled occupation and presence of DM complications were the measurable risk factors in development of cognitive impairment.
In this study, the prevalence of cognitive impairment in DM patients is seemingly high with both scores i.e. $96.31 \%$ with MoCA and $99.85 \%$ with ACE$\mathrm{R}$ respectively. Cognitive impairment is high in older population, as almost all in this groups have cognitive impairment. Similar high prevalence was noted by earlier studies of S. C. Tiwari et $\mathrm{al}^{15}$ Moreover cognitive impairment and dementia was found to be high in patient of having age group 45 to 60 years (28.32\% and $34.81 \%$ respectively) than in age group of $>60$ years $(13.13 \%$ and $16.67 \%$ respectively). This may be because of DM which contributes to higher $\mathrm{CI}$ in this young adult population may be due duration of diabetes or the more number of study participant were from young adult group i.e. 45 to 60 years $(68.29 \%)$.

Various studies stated in relation to cognitive impairment and DM is inconclusive because of inconsistent reports. The variation in findings may be due to differences in the study design, sources of study subjects, and variations in criteria for the diagnosis of DM or cognitive impairment. Likewise, it may also be due to differences in the duration or severity of DM among study subjects. ${ }^{16}$ Hassing et al showed that, the association between DM and cognitive impairment and older adults with DM experience global cognitive have more decline in rate compared to those who without DM. ${ }^{17}$ Literature claimed that the average prevalence of MCI in DM ranging from $20 \%$ to $38 \%$. Whereas in present study we found that MCI was seen in a relatively high proportion 299 (43.21\%). The incidence is very high as compared to other studies in literature. The reason for higher incidence could also be because this study was done in DM patients who attending neurology Out Patient Department (OPD).

Most of the studies showed that, females were more susceptible to occurrence of dementia. This study also showed the same pattern i.e. higher number of females $(26.84 \%)$ than males $(25.36 \%)$. However, males $(37.7 \%)$ were more susceptible to MCI than female $(6.3 \%)$. while in case of occupation, we found that, housewives $(27.1 \%)$ were more susceptible to dementia and service personnel's (20.2\%) were more susceptible to MCI. As the dementia is more severe form of cognitive impairment, leads to $\mathrm{AD}$. With our result it was seen that the more education leads to less complications in cognitive impairment due to the DM..$^{18}$

However, this data was not statistically significant when compared the susceptibility of gender with the occurrence of the cognitive impairment.

In the present study, MCI detected with both tests was inversely associated with years of education, as expected and shown in many previous studies, cognitive tests scores herein were superior in those individuals that were more educated. ${ }^{19}$

Low education attainment was also be one of the contributing factors and was associated with increased prevalence of cognitive impairment. ${ }^{20,21}$ Moreover, illiterates are usually more tangled in unhealthy 
lifestyle practices like smoking and excessive use of alcoholic drinks. Besides, they may be involved in unhealthy feeding habits and may not have access to adequate medical care. ${ }^{22}$

Cognitive impairment was found to be is higher and significant in housewives, as compared to service, farmer, and business class. This is alike to several other studies. Unskilled occupation like farmworker farming or other physical works was associated with higher prevalence of cognitive impairment. This is because those involved in uneducated occupation are mainly illiterates. They did not have the privilege of formal education or could not deal with the rigors of formal education and of skilled occupation. All domains of MoCA have shown significant difference between patients with and without cognitive impairment excluding memory which scored zero in both. This showed that there was severe cognitive impairment regarding all domains. ${ }^{23}$

Alcohol consumption is one promising menace cause for dementia. Alcoholism is allied with extensive cognitive problems, including alcoholic dementia. Because alcohol's effects on cognition, brain disorders, and brain chemistry contribute to some features with AD's effects on these three areas, it is likely that alcohol use might also increase the risk of developing $\mathrm{AD}^{23}$
Olivia Lerche et al pointed that, alcohol consumption is accompanying with an increased risk of adverse brain consequences and steeper decline in cognitive abilities. ${ }^{24}$ The Experts personnel's from the University of Oxford and University College London studied 550 people over a 30-year period, constantly assessing their alcohol consumption and cognition. They find that, usage of alcohol was lead to the reduced right hippocampal volume - oart of the brain a form of brain damage that affects memory and spatial navigation. ${ }^{25}$ Interestingly, in present study; we did not find any significant effect of alcohol addiction on the dementia in DM patient.

Various short screening tests, such as MoCA, Mini-Cog, or MMSE, ACE are useful to assess overall cognition; MoCA is available in the public domain. The Mini-Cog is a short screening tool that is found to be useful in a busy clinical practice to screen for cognitive dysfunction including executive dysfunction. ${ }^{26}$

Based on the current evidences we can conclude that, the occurrence of accelerated cognitive dementia is might associated with DM or old age or both DM and old age. It is a complex mechanism and should more research is needed to clarify the neurobiological mechanism involved in cognitive impairment and DM.

Table 1: The various socio-demographic variables with their MoCA score and Addenbrooke's score

\begin{tabular}{|c|c|c|c|c|c|}
\hline \multirow{2}{*}{$\begin{array}{c}\text { Socio-Demographic } \\
\text { Variables }\end{array}$} & \multicolumn{3}{|c|}{ MoCA Score } & \multicolumn{2}{|c|}{ ACE-R score } \\
\hline & $\begin{array}{c}<18 \\
\text { (Dementia) } \\
\mathbf{N}(\%)\end{array}$ & $\begin{array}{c}\mathbf{1 8 - 2 5} \\
\text { (MCI.) } \\
\text { N }(\%)\end{array}$ & $\begin{array}{c}\geq 26 \\
N(\%)\end{array}$ & $\begin{array}{c}\text { less than } 83 \\
\text { MCI } \\
\text { N }(\%)\end{array}$ & $\begin{array}{c}\text { More } \\
\text { than } 83 \\
\mathrm{~N}(\%)\end{array}$ \\
\hline \multicolumn{6}{|l|}{ Age group } \\
\hline $45-60$ & $236(34.81)$ & $192(28.32)$ & $36(5.31)$ & $463(68.29)$ & $1(0.14)$ \\
\hline$>60$ & $113(16.67)$ & $89(13.13)$ & $12(1.77)$ & $214(31.56)$ & 0 \\
\hline \multicolumn{6}{|l|}{ Sex } \\
\hline Female & $182(26.84)$ & $43(6.3)$ & $4(0.5)$ & $229(33.78)$ & $0(0)$ \\
\hline Male & $172(25.36)$ & $256(37.7)$ & $21(3.09)$ & $448(66.07)$ & $1(0.147)$ \\
\hline \multicolumn{6}{|l|}{ Occupation } \\
\hline Business & $62(9.1)$ & $86(12.6)$ & $4(0.5)$ & $152(22.41)$ & $0(0)$ \\
\hline Farmer & $53(7.8)$ & $40(5.89)$ & $0(0)$ & $93(13.71)$ & $0(0)$ \\
\hline Housewife & $184(27.1)$ & $36(5.3)$ & $2(0.2)$ & $222(32.74)$ & 0 \\
\hline Service & $55(8.1)$ & $137(20.2)$ & $19(2.8)$ & $210(30.97)$ & $1(0.14)$ \\
\hline \multicolumn{6}{|l|}{ Education } \\
\hline Illiterate & $171(25.2)$ & $9(1.3)$ & $0(0)$ & $180(26.54)$ & $0(0)$ \\
\hline Primary & $96(14.1)$ & $50(7.3)$ & $0(0)$ & $146(21.53)$ & $0(0)$ \\
\hline SSC & $26(3.8)$ & $63(9.2)$ & $2(0.2)$ & $91(13.42)$ & $0(0)$ \\
\hline HSC & $28(4.1)$ & 71(10.4) & $8(1.17)$ & 107(15.78) & $0(0)$ \\
\hline Degree & $23(3.3)$ & $69(10.17)$ & $7(1.03)$ & $98(14.45)$ & $1(0.14)$ \\
\hline Post Graduate $\&$ above & $10(1.4)$ & $37(5.45)$ & $8(1.17)$ & $55(8.11)$ & $0(0)$ \\
\hline \multicolumn{6}{|l|}{ Alcohol Addiction } \\
\hline Yes & $124(18.28)$ & $158(23.3)$ & $8(1.17)$ & $290(42.77)$ & $0(0)$ \\
\hline No & $230(33.9)$ & $141(20.7)$ & $17(2.5)$ & $387(57.07)$ & $1(0.14)$ \\
\hline
\end{tabular}

Footnote: Data are presented as frequency and percent (\%), as appropriate. MoCA: Montreal Cognitive Assessment; ACE: Addenbrooke's Cognitive Examination, SSC: Secondary School Certificate; HSC: Higher secondary school. 
Table 2: Comparison of different items in MoCA score in diabetic patients

\begin{tabular}{|l|c|c|c|c|c|}
\hline $\begin{array}{c}\text { MoCA domain } \\
\text { Median score } \\
\text { (range) }\end{array}$ & $\begin{array}{c}<\mathbf{1 8} \\
\text { (Dementia) }\end{array}$ & $\begin{array}{c}\mathbf{1 8 - 2 5} \\
(\mathbf{M C I})\end{array}$ & $\mathbf{2 6}$ & $\mathbf{P}$-value & $\begin{array}{c}\text { Significant } \\
\text { at 5\% level }\end{array}$ \\
\hline $\mathrm{N}$ & 354 & 299 & 25 & & \\
\hline Visuo-excutive/5 & $1(0-4)$ & $3(0-5)$ & $5(3-5)$ & $<0.001^{* * * *}$ & Yes \\
\hline Naming/3 & $2(0-3)$ & $3(0-3)$ & $3(2-3)$ & $<0.001^{* * * *}$ & Yes \\
\hline Memory/5 & $0(0-0)$ & $0(0-2)$ & $0(0-0)$ & $0.531^{\text {ns }}$ & No \\
\hline Attention/6 & $3(0-5)$ & $5(3-6)$ & $6(4-6)$ & $<0.001^{* * * *}$ & Yes \\
\hline Language/3 & $3(0-3)$ & $3(2-6)$ & $3(3-3)$ & $<0.001^{* * * *}$ & Yes \\
\hline Abstraction/2 & $0(0-2)$ & $1(0-3)$ & $1(1-2)$ & $<0.001^{* * * *}$ & Yes \\
\hline Orientation/6 & $4(0-6)$ & $6(2-6)$ & $6(6-6)$ & $<0.001^{* * * *}$ & Yes \\
\hline
\end{tabular}

Footnote: MoCA: Montreal Cognitive Assessment; MCI: Mild cognitive impairment. A p value of $<0.05$ was considered as statistically significant.

\section{Conclusion}

A clinician needs to recognize different domains of cognition affecting the patients with DM to understand which self-care behavior will be affected in that individual. Traditional DM management strategies stress the role of the patients an important member of the DM management team and focus on the self-care education needed to care for DM and related syndromes. Thus, the presence of cognitive dysfunction (also commonly referred to as $\mathrm{MCI}$ ) is an important condition to recognize as it interferes with patients' participation in their DM management. Hence, the identification of DM -related dementia or elder age related dementia may be necessary for considering an appropriate therapy and prevention in clinical practice.

\section{Limitations}

It is a simple retrospective cohort study and not has a sufficient control treatment group like non-diabetic patients. It was also noticed that the sample of cognitive impairment subjects is small to foresee robust association.

\section{Acknowledgment: None}

\section{References}

1. International Diabetes Federation. IDF Diabetes Atlas. 8th edition. Brussels, Belgium: International Diabetes Federation; 2017.

2. Census of India. Rural urban distribution of population. Office of the registrar general and census commissioner, India. Available from: http://censusindia.gov.in/2011prov-results/ paper2/ data_files/India/ Rural_Urban_2011.pdf. (Accessed on 7 Nov 2016)

3. https://www.firstpost.com/india/diabetes-is-indias-fastestgrowing-disease-72-million-cases-recorded-in-2017figure-expected-to-nearly-double-by-2025-4435203.html

4. M. Stumvoll, B. J. Goldstein, and T. W. van Haeften. Type 2 diabetes: principles of pathogenesis and therapy. The Lancet 2005;365:1333-46.

5. J. A. Luchsinger, C. Reitz, B. Patel, M.-X. Tang, J. J. Manly, and R. Mayeux. Relation of diabetes to mild cognitive impairment. Arch Neurol 2007;64:570-75.

6. Barbagallo M, Dominguez LJ. Type 2 diabetes mellitus and Alzheimer's disease. World J Diabetes 2014;5:88993.
7. Li L, Wang Y, Yan J, et al. Clinical predictors of cognitive decline in patients with mild cognitive impairment: the Chongqing aging study. J Neurol 2012;259:1303-11.

8. Barbagallo M, Dominguez LJ. Type 2 diabetes mellitus and Alzheimer's disease. World J Diabetes 2014;5:88993.

9. Tuba Özcan1and Esra Yancar Demir. Investigation the Cognitive Impairment in Diabetes Mellitus Type 2 with Moca. J Psychiatry 2014;17:6. 1000166. doi:10.4172/Psychiatry.1000166.

10. P. S. Singh*, Himanshu Sharma, Khwaja S. Zafar, Prafulla K. Singh, Sudhir K. Yadav, Rajesh K. Gautam, Tony Pious. Prevalence of type 2 diabetes mellitus in rural population of Indiaa study from Western Uttar Pradesh. Int J Res Med Sci 2017;5(4):1363-67.

11. C. T. Kodl and E. R. Seaquist. Cognitive dysfunction and diabetes mellitus. Endocrine Reviews 2008;29:494-11.

12. Nasreddine ZS, Phillips NA, Bédirian V, et al. The Montreal Cognitive Assessment, MoCA: a brief screening tool for mild cognitive impairment. $J A m$ Geriatr Soc 2005;53:695-99.

13. Shuba N, Karan. Assessment of the Cognitive Status in Diabetes Mellitus. J Clin Diagnostic Res 2012;6(10):1658-62.

14. Eze CO, Ezeokpo BC, Kalu UA, Onwuekwe IO. The Prevalence of Cognitive Impairment amongst Type 2 Diabetes Mellitus Patients at Abakaliki South-East Nigeria. J Metabolic Synd 2015;4:171. doi:10.4172/21670943.1000171.

15. S. C. Tiwari, Rakesh Kumar Tripathi, Siddiq Ahmad Farooqi, Rajesh Kumar, Garima Srivastava, and Aditya Kumar. Diabetes mellitus: A risk factor for cognitive impairment amongst urban older adults. Ind Psychiatry $J$ 2012;21(1):44-8.

16. Tiwari SC, Kar AM, Singh R, Kohli VK, Agarwal GG. Final report submitted to Indian Council of Medical Research. New Delhi, India: 2009. An epidemiological study of prevalence of neuro-psychiatric disorders with special reference to cognitive disorders amongst urban elderly.

17. Linda B. Hassing, Michael D. Grant, Scott M. Hofer, Nancy L. Pedersen, Sven E. Nilsson, Stig Berg, Gerald Mcclearn, and Boo Johansson. Type 2 diabetes mellitus contributes to cognitive decline in old age: A longitudinal population-based study. J Int Neuropsychol Soc 2004;10:599-607.

18. Forlenza et al.: Diagnosis and biomarkers of predementia in Alzheimer's disease. BMC Med 2010;8:89. 
19. Ferreira L, Ferreira Santos-Galduroz R, Ferri CP, Fernandes Galduroz JC. Rate of cognitive decline in relation to sex after 60 years-of-age: a systematic review. Geriatrics Gerontol Int (2014);14(1):23-31.

20. Sharp ES, Gatz M. Relationship between education and dementia: an updated systematic review. Alzheimer disease and associated disorders 2011;5(4):289-04.

21. Callahan CM, Hall KS, Hui SL, Musick BS, Unverzagt FW, Hendrie HC at el. Relationship of age, education, and occupation with dementia among a community-based sample of African Americans. Arch Neurol 1996;53(2):134-40.

22. Evert, D.L., and Oscar-Berman, M. Alcohol-related cognitive impairments: An overview of how alcoholism may affect the workings of the brain. Alcohol Health \& Research World 1995;19(2):89-6.
23. Smith, D.M., and Atkinson, R.M. Alcoholism and dementia. In: Gurnack, A.M., ed. Older Adults' Misuse of Alcohol, Medicines, and Other Drugs: Research and Practice Issues. New York: Springer, 1997:132-57.

24. Olivia lerche. Dementia and alcohol link revealed: You should only be drinking THIS

much.https://www.express.co.uk/lifestyle/health/814023/dementa-symptoms-alzheimers-linkalcohol-units.

25. Bate C, Williams A. (2011). Ethanol protects cultured neurons against amyloid-beta and alpha-synucleininduced synapse damage. Neuropharmacol 2011;61:1406-12.

26. Medha N. Munshi Cognitive Dysfunction in Older Adults With Diabetes: What a Clinician Needs to Know. Diabetes Care 2017;40:461-67. 